\title{
Biography—Li Ma
}

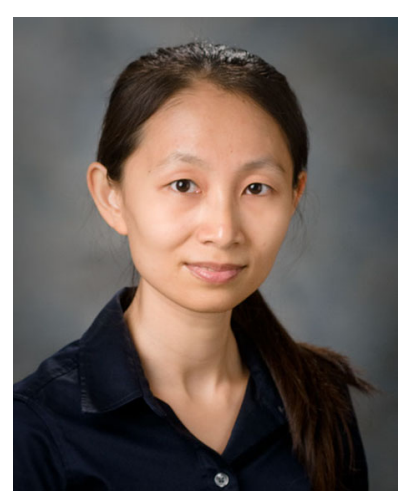

Dr. Li Ma is an Associate Professor in the Department of Experimental Radiation Oncology at The University of Texas MD Anderson Cancer Center. Dr. Ma received her bachelor's degree in biology from Peking University in 2001. After completing her Ph.D. study at Sloan-Kettering Institute and Cornell University Weill Graduate School of Medical Sciences in 2006, she became a postdoctoral fellow at the Whitehead Institute for Biomedical Research. She joined the faculty of MD Anderson as an Assistant Professor in 2010 and was promoted to Associate Professor with tenure in 2015.

Dr. Ma studies tumor metastasis, therapy resistance, and regulation of key cancer proteins and pathways. Her interests include noncoding RNAs, developmental regulators, and deubiquitinating enzymes. She published original research in leading journals including Cell, Nature, Nature Medicine, Nature Cell Biology, and Nature Biotechnology, with high volumes of citations. She has been recognized by prestigious national and international awards, including the 2010 CPRIT Scholar Award, the 2014 AAAS Martin and Rose Wachtel Cancer Research Award, and the 2016 AACR Stand Up to Cancer Innovative Research Award. Currently, she is the Principal Investigator of two NCI R01 grants, a CPRIT research grant, and an AACR SU2C grant. Dr. Ma is an Editorial Board member of Science Signaling. She has served on many review panels, including NCI's P01, R01, R21, and K study sections. 